\title{
Análise de conteúdo aos diagnósticos e intervenções tegumentares: 0 primeiro passo para um modelo de cicatrização de feridas
}

\author{
João Gomes ${ }^{1,2}$, Paulino Sousa ${ }^{2,3}$, Filipe Pereira ${ }^{2,3}$, Fernanda Bastos ${ }^{2,5}$ \\ Ana Paula Prata ${ }^{2,5}$ e Catarina Silva ${ }^{6}$ \\ 1 Doutorando, Instituto de Ciências Biomédicas de Abel Salazar, Portugal. Enfermeiro, Hospital de \\ Braga, Portugal | enfermeirojoao@gmail.com | https://orcid.org/0000-0001-5060-7976 \\ ${ }^{2}$ Centro de Investigação Desenvolvimento em Sistemas de Informação em Enfermagem da Escola \\ Superior de Enfermagem do Porto, Portugal (CIDESI-ESEP) \\ ${ }^{3}$ Professor Coordenador, Escola Superior de Enfermagem do Porto, Portugal | paulino@esenf.pt; \\ filipereira@esenf.pt | https://orcid.org/0000-0002-5778-0111; https://orcid.org/0000-0000-0000-0000 \\ ${ }^{5}$ Professor Associado, Escola Superior de Enfermagem do Porto, Portugal \\ fernandabastos@esenf.pt; prata@esenf.pt | https://orcid.org/0000-0003-3097-7114; \\ https://orcid.org/0000-0001-7552-9716 \\ ${ }^{6}$ Enfermeira Especialista, ACES Alto Ave, Portugal | catsilva@gmail.com | https://orcid.org/0000- \\ 0002-7597-9343
}

Resumo: Introdução: Especialistas em tratamento de feridas são essenciais para abordar necessidades crescentes de prevenção e tratamento de feridas que afetam milhões de pessoas. Os sistemas de informação com apoio à tomada de decisão clínica tornam-se essenciais ao recolher, codificar e armazenar conhecimento, devolvendo informação pertinente ao enfermeiro. Objetivos: Este estudo visa identificar oportunidades para melhoria da interoperabilidade semântica através da análise da construção e conceitos presentes nos diagnósticos e intervenções do Sistema de Informação em Saúde. Métodos: Estudo descritivo com recolha de dados no Sistema de Informação em Saúde Português, sendo reconhecido pelo progresso em termos de tecnologias de informação e comunicação no setor da saúde. Todos os diagnósticos, intervenções e relações disponíveis para a prática clínica no âmbito do processo tegumentar foram extraídos. Realizámos esta investigação em duas fases: (1) Análise de conteúdo aos diagnósticos e intervenções, revista por dois peritos externos e (2) Grupo focal com peritos em Sistemas de Informação em Saúde de forma a discutir os resultados anteriores $e$ implementar contribuições para um modelo clínico relativo à cicatrização de feridas. Resultados: Fizemos correspondência a 66 diagnósticos, 171 intervenções e 318 relações. As intervenções de enfermagem são centradas em três tipos de ação: executar, determinar e informar. Encontrámos inconsistências inesperadas no que diz respeito às associações entre ações do tipo ensinar e a ausência de diagnósticos centrados no conhecimento. O grupo focal acrescentou uma importante perspetiva na importância de intervenções de outros domínios para a cicatrização de feridas. Conclusões: Os enfermeiros usam uma linguagem padronizada para descrever os conceitos na prática clínica. A interoperabilidade semântica é questionável por alterações sustentadas em opiniões no lugar de conhecimento científico, sem normas ou procedimentos de domínio público. A abordagem qualitativa permitiu melhorias em termos dos conceitos utilizados para representar a prática clínica e validade da construção dos enunciados.

Palavras-chave: Modelo Clínico de Informação; Diagnóstico de Enfermagem; Intervenção de Enfermagem; Cicatrização; Ferimentos e Lesões.

Content Analysis of Nursing Integumentary Diagnoses and Interventions: Firsts Step Towards a Wound Healing Information Mode

Abstract: Introduction: Wound care specialists are essential to meet the growing need to prevent and treat acute and chronic wounds affecting millions worldwide. Information systems with clinical decision support become essential by collecting, codifying, and storing knowledge, and returning information onscreen to the nurse.Goals: This study aims to identify opportunities for semantic interoperability improvement through the analysis of all diagnoses and interventions used in a public Health Information System related to the integumentary system. Methods: A qualitative descriptive study of nursing diagnoses and interventions data. We selected the Portuguese national Health Information System for data analysis since it was recognized for the progress in terms of information and communications technologies used in the health sector. All nursing diagnoses, interventions, and their relationships available for selection in clinical practice regarding wound healing were analyzed. We performed this research in two phases: (1) Content analysis of nursing diagnoses and interventions, revised by two external experts, and (2) Focus group with Health Information Systems and terminologies experts to discuss and implement contributions to a wound healing clinical model.Results: We matched 66 diagnoses, 171 interventions, and 318 relationships to the integumentary system. Nursing interventions were centered on three action types: performing, determining, and informing. We found unexpected inconsistencies regarding the association between teaching actions and missing knowledge-centered diagnoses. The focus group added an essential perspective on interventions from other domains becoming essential to wound healing. Conclusions: Nurses use standardized language to describe nursing concepts in daily practice. Semantic interoperability is still questionable by persistent changes based on opinions instead of scientific knowledge, with no known public rules or procedures. A qualitative approach allowed for improvements in the concepts used to represent clinical practice and the sentences construction validity.

Keywords: Clinical Information Model; Nursing Diagnosis; Nursing Intervention; Wound Healing Wounds and Injuries. 


\section{Introdução}

Desde que Nightingale abriu caminho para a enfermagem moderna ao cuidar de soldados e prevenindo infeções e mortes causadas por feridas, mais de 150 anos de conhecimento científico acumularam no domínio da cicatrização (Strickler, 2017). No nosso século, as preocupações estão a inverter de feridas traumáticas para um número exponencial de doenças crónicas, com as feridas de difícil cicatrização a colocarem um peso significativo nos sistemas de saúde, nas pessoas que vivem com feridas e na sua família (Woo et al., 2018). Dados europeus sugerem que $64 \%$ das feridas tratadas em casa são crónicas e a gestão de feridas ocupa cerca de $3 \%$ de todas as despesas de saúde (Lindholm \& Searle, 2016). O custo por tratamento varia no entanto com fatores como o tipo de ferida, complicações ou a instituição (Nussbaum et al., 2018).

Especialistas em tratamento de feridas são necessários para ir de encontro à necessidade crescente de prevenir e tratar feridas agudas e crónicas. Os enfermeiros providenciam cuidados à pessoa com um leque alargado de feridas causadas por pressão, insuficiência venosa, arterial, diabetes, trauma ou cirurgias. As feridas podem ter efeitos devastadores sobre a pessoa aumentando a morbilidade e mortalidade associadas a complicações. A dor, sangramento, exsudado, necrose, infeção ou amputação são algumas destas complicações (WOCN Task Force, 2018).

Criar um ambiente favorável à cicatrização é um processo complexo que requer coordenação de decisões e ações em resposta a várias condições. Tomar decisões e realizar juízos clínicos na avaliação e planeamento de cuidados são um desafio que os enfermeiros enfrentam por falta de evidência sólida (Samuriwo \& Dowding, 2014).

A documentação de cuidados tem várias lacunas, causando constrangimentos no futuro do conhecimento de enfermagem e desenvolvimento de uma identidade profissional. Em alguns trabalhos, foi evidenciado que $50 \%$ das pessoas tratadas não têm um diagnóstico adequado documentado (Ivins et al., 2018) e a troca de apósitos, bem como a documentação da avaliação, não é realizada, dificultando a análise dos resultados (Batista da Cunha et al., 2018).

Os termos e conceitos utilizados também necessitam de consenso para os profissionais estabelecerem melhores critérios diagnósticos, eliminando variações não desejadas da tomada de decisão e providenciando melhores cuidados através de procedimentos padronizados (Atkin \& Tickle, 2018). A Classificação Internacional para Prática de Enfermagem (CIPE) é uma terminologia de enfermagem reconhecida pela Organização Mundial de Saúde (2017). As terminologias são representações de conceitos clínicos necessárias para descrever condições e atividades (International Organization for Standardization, 2014). A utilização destes vocabulários controlados é recomendada para a representação de conceitos e relações dado que permitem uma comunicação clara de informação clínica entre remetente e recetor, local ou internacionalmente (Lozano-Rubí et al., 2016).

A utilização de terminologias em modelos clínicos melhora sistematicamente a interoperabilidade semântica, definida como "assegurar que o significado preciso da informação comutada é percetível por qualquer outro sistema ou aplicação não desenvolvido inicialmente para esse propósito" (International Organization for Standardization, 2015, p.5). Esta capacidade de permutar informação entres diferentes sistemas sem perda de significado permite uma implementação segura e efetiva do suporte à tomada de decisão.

Este artigo descreve a primeira fase na direção deste objetivo, consistindo numa análise de conteúdo focada no sistema tegumentar. Este estudo visa identificar as oportunidades para a melhoria da interoperabilidade semântica através de uma análise da construção e dos conceitos presentes nos diagnósticos e intervenções em uso no Sistema de Informação em Saúde (SIS) português. 


\section{Metodologia}

A documentação de enfermagem em Portugal começou a migrar em 2005 de suportes em papel para registos eletrónicos. Cada instituição foi convidada a desenvolver os seus conjuntos de diagnósticos e intervenções com recurso à CIPE, sendo que cada um destes agrupamentos foi descrito como uma "parametrização". Ao longo de uma década foram criadas centenas de parametrizações nas várias instituições do país, com diferentes nomenclaturas para representar os mesmos conceitos, o que colocou um compromisso severo na interoperabilidade semântica e na mensuração de indicadores (Paiva et al., 2014).

A Escola Superior de Enfermagem do Porto (ESEP) analisou em 2014 todas as parametrizações em uso e propôs uma parametrização única para todas as instituições, implementada depois pelo Ministério da Saúde (Paiva et al., 2014). Após este esfoço, o Centro de Investigação Desenvolvimento em Sistemas de Informação em Enfermagem da Escola Superior de Enfermagem do Porto (CIDESI-ESEP) decidiu criar uma ontologia de enfermagem, definida como uma descrição de conceitos e relações de um domínio científico (Gruber, 1993). Esta ontologia, desenvolvida em parceria com a Ordem dos Enfermeiros que tem um papel regulatório no exercício profissional (Ordem dos Enfermeiros, 2018), é constituída por vários modelos de informação em diversas áreas. Cruz et al. (2016), Gonçalves et al. (2019), Neves e Parente (2019) e Queirós et al. (2021) desenvolveram trabalhos iniciais nas áreas do regime medicamentoso, saúde mental, processo neuromuscular e autocuidado, respetivamente.

Um estudo descritivo e exploratório foi agora desenvolvido para analisar os diagnósticos e intervenções de enfermagem parametrizados no SIS relativos ao sistema tegumentar. Um diagnóstico de enfermagem é um rótulo atribuído a um achado de uma observação ou evento que é considerado relevante para o enfermeiro e a pessoa cuidada. Por sua vez, uma intervenção de enfermagem é uma ação implementada para melhorar ou manter a saúde uma pessoa, grupo ou população (International Organization for Standardization, 2014).

Foram seguidas as recomendações da ISO 13972 que orientam para a pesquisa dos conteúdos existentes nos sistemas de informação em uso e do envolvimento dos peritos no domínio científico na criação de modelos de dados.

Consiste em duas fases sequenciais:

(1) Análise de conteúdo aos diagnósticos e intervenções de enfermagem extraídos do SIS em uso no Sistema Nacional de Saúde português, revista por dois peritos externos, e

(2) Grupo focal com quatorze enfermeiros, peritos em desenvolvimento de conteúdos para os SIS com recurso a terminologias de enfermagem, de forma a discutir os resultados obtidos na fase anterior e implementar novas contribuições para um modelo de informação.

A aprovação deste projeto foi atribuída pela comissão de ética da ESEP (ADHOC_553/2020). Apenas os focos, diagnósticos e intervenções, bem como as relações entre eles, foram extraídos e analisados neste projeto (por exemplo, "ferida cirúrgica" ou "Avaliar queimadura"). Iremos referir-nos a estas concatenações de termos como "frases" que os enfermeiros usaram para descrever a sua prática clínica.

Dado que apenas analisámos uma parametrização em vez de registos clínicos descritivos, não foram recolhidos dados relativos à pessoa, enfermeiro, instituição ou data, garantindo a anonimização e todos os direitos relativos à proteção de dados. 


\subsection{Fase 1 - Análise de Conteúdo aos Diagnósticos e Intervenções de Enfermagem}

De acordo com Bardin (1977), seguimos um método de análise de conteúdo para atingir um conjunto de categorias que representem a prática de enfermagem, minimizando a ocorrência de múltiplas frases representando o mesmo conceito clínico.

Um enfermeiro com grau de mestre, doutorando em Ciências de Enfermagem, pósgraduado em sistemas de informação em enfermagem e em intervenção avançada em feridas realizou esta fase. Dois enfermeiros doutorados em Ciências de Enfermagem com mais de quinze anos de experiência no desenvolvimento e análise de conteúdos para os SIS realizaram a revisão da análise.

\subsubsection{Pré-análise}

Selecionámos o SIS público português (SClínico $\AA$ ) para extração dos dados dado que Portugal é reconhecido pelo progresso em tecnologias de informação e comunicação na área da saúde (European Observatory on Health Systems and Policies, 2018). A maioria dos serviços públicos usam este SIS e os seus conteúdos estão em contínuo desenvolvimento há mais de 20 anos. É usado em contexto hospitalar e comunitário.

Este SIS permite o registo descritivo dos cuidados, mas tem por suporte a parametrização única proposta pela ESEP, agora mantida e atualizada pelo Ministério da Saúde. Esta parametrização é constituída por frases pré-concatenadas de diagnósticos, intervenções e relações entre eles tendo por suporte a terminologia da CIPE. Dado o âmbito alargado desta parametrização, a sua aplicação na maioria das instituições e o desenvolvimento contínuo ao longo de décadas, consideramos a nossa amostra representativa dos cuidados de enfermagem.

Todos os focos, diagnósticos, intervenções e relações acessíveis em maio de 2016 foram disponibilizados pelos Serviços Partilhados do Ministério da Saúde à ESEP. Foi realizada uma leitura flutuante de todos os focos existentes na parametrização e selecionámos os relevantes para o sistema tegumentar, extraindo para uma base de dados. Utilizámos a definição da CIPE (2017) para o sistema tegumentar, que envolve a pele, epiderme, derme, mucosa, tecido subcutâneo, dermas, glândulas sebáceas, cabelo e unhas.

Após selecionar todos os focos, extraímos os diagnósticos e intervenções associados a eles, bem como as relações existentes. Foi utilizado outro critério de inclusão relativo à pessoa cuidada: apenas as frases relativas à própria pessoa foram extraídas, desconsiderando todos os conteúdos relativos a cuidadores ou pais.

\subsubsection{Codificação}

Utilizámos a CIPE (2017) como terminologia de forma a obter os conceitos que sustentam o enunciado e a norma ISO 18104:2014 para analisar a validade das construções das frases de diagnósticos e intervenções, atualizando as mesmas sempre que possível e criando o corpo de análise.

Todas as frases extraídas estavam num formato legível com estrutura semântica adequada, pelo que não foi necessária uma análise do contexto das mesmas antes do processo indutivo.

\subsubsection{Categorização e Interpretação}

Não utilizámos categorias definidas a priori. Cada frase foi usada como unidade de registo para análise, depois agregada e organizada em categorias através de um processo indutivo. Tentámos promover a consistência entre diferentes níveis de granularidade presentes nas frases para atingir o melhor potencial para a interoperabilidade semântica. 
Após categorização de todo o corpo de análise, as categorias que emergiram foram revistas por dois peritos externos para discussão e reavaliação do processo.

\subsection{Fase 2 - Grupo Focal}

Dada a natureza qualitativa do processo de análise, um grupo focal foi selecionado como método para discussão dos resultados anteriores através da exposição dos critérios de categorização sustentados na terminologia e nas regras de construção dos enunciados. Como apresentado por Itulua-Abumere (2015), um grupo focal consegue desmascarar ideias, crenças e opiniões originadas pela análise qualitativa e esclarece alguns malentendidos com frases que necessitam de clarificação. É também uma abordagem rápida para a análise de grandes quantidades de informação e providencia uma oportunidade para discutir várias perspetivas sempre que necessário.

Cada unidade de registo foi apresentada ao grupo com uma categoria proposta ou um critério de exclusão. Seguiu-se uma breve discussão onde os participantes puderam sugerir outras opiniões. Se não for atingido consenso em determinado assunto, decidimos incluir e categorizar a frase.

Mais do que repetir a fase anterior com um grupo maior, nesta reunião promoveu-se a discussão de critérios utilizados em relação a outros modelos de informação em desenvolvimento na ontologia. Novas ideias foram discutidas e as relações com outros focos foram exploradas.

\section{Resultados}

\subsection{Fase 1}

Após analisar os focos extraídos, fizemos a correspondência de 18 ao sistema tegumentar, extraindo depois todos os diagnósticos, intervenções e relações entre eles, criando uma lista com 18 focos, 66 diagnósticos, 171 intervenções diferentes e 318 relações, resumidos na tabela 1.

Tabela 1. Relações verificadas entre os focos, diagnósticos e intervenções de enfermagem

\begin{tabular}{lcc}
\hline \multicolumn{1}{c}{ Focos } & Diagnósticos & Intervenções associadas ao diagnóstico \\
\hline Candidíase & 3 & 9 \\
Dor Por Ferida & 5 & 32 \\
Eritema & 3 & 9 \\
Eritema Pelas Fraldas & 3 & 9 \\
Escoriação & 1 & 3 \\
Exantema & 1 & 4 \\
Ferida & 6 & 29 \\
Ferida Cirúrgica & 3 & 15 \\
Ferida Traumática & 3 & 25 \\
Fissura & 3 & 11 \\
Laceração & 1 & 14 \\
Maceração & 4 & 22 \\
Membrana Mucosa & 3 & 7 \\
Queimadura & 4 & 13 \\
\hline
\end{tabular}




\begin{tabular}{lcc}
\multicolumn{1}{c}{ Focos } & Diagnósticos & Intervenções associadas ao diagnóstico \\
\hline Úlcera & 9 & 48 \\
Úlcera Arterial & 3 & 8 \\
Úlcera por Pressão & 7 & 43 \\
Úlcera Venosa & 4 & 17 \\
Total & 66 & $\mathbf{3 1 8}$ \\
\hline
\end{tabular}

Realizámos a análise de todas as frases extraídas relativamente ao processo de construção pela norma ISO 18104:2014, tendo em conta o objetivo de cicatrização para cada diagnóstico, resultando na exclusão de 46 diagnósticos e 90 intervenções. Isto representa uma exclusão de $70 \%$ dos diagnósticos e $50 \%$ das intervenções para prevenir redundâncias no sistema de informação, dado representarem outras áreas de atenção como a capacidade para se posicionar de forma a prevenir por exemplo uma úlcera de pressão. Outros diagnósticos foram excluídos por não terem um juízo clínico associado ao foco ou por terem um conceito clínico demasiado abstrato.

As restantes frases foram depois analisadas qualitativamente para criar categorias. Para a maioria utilizámos uma categorização in vivo com única frase como amostra. Esta singularidade demostra um bom nível de otimização da linguagem para a promoção da interoperabilidade semântica. Algumas frases envolvendo graus, como no caso das queimaduras, foram agrupadas numa única categoria dado que especificação destes graus corresponde à avaliação de dados realizada após o diagnóstico ser identificado.

Analisando a estrutura dos diagnósticos pela normal ISO 18104:2014, verificámos que a maioria são achados clínicos como "Úlcera por Pressão", conceitos que não necessitam de um juízo na sua construção, em oposição a outros conceitos que dependem de campos adicionais como a oportunidade e o juízo, como o "Potencial para melhorar o conhecimento sobre a cicatrização da úlcera arterial".

As categorias das intervenções também foram criadas essencialmente in vivo, excetuando algumas ações do tipo avaliar onde várias frases tinham como alvo as características da ferida. As categorias criadas são centradas em três tipos de ação: executar, determinar e informar, o que em termos práticos significa avaliar a ferida, tratá-la e ensinar sobre a cicatrização.

Terminámos a análise com a leitura de todas as relações entre diagnósticos e relações, resultado em 247 exclusões, essencialmente pelos diagnósticos ou intervenções previamente excluídos, retirando o contexto à relação. Seis associações foram consideradas inadequadas por não existir relação lógica ou científica entre elas, como "Ferida traumática - Aplicar terapia compressiva", dado que esta intervenção é clinicamente indicada na cicatrização de úlceras venosas.

As restantes 71 relações foram agrupadas por foco e diagnóstico na tabela 2. Esta representa a primeira versão do modelo de dados, baseada nas frases parametrizadas pelos enfermeiros para uso no SIS no que diz respeito ao processo tegumentar.

Tabela 2. Relações entre focos, diagnósticos e intervenções

\begin{tabular}{lll}
\hline \multicolumn{1}{c}{ Focos } & Diagnósticos & Intervenções \\
\hline $\begin{array}{ll}\text { Eritema pelas } \\
\text { Fraldas }\end{array}$ & $\begin{array}{l}\text { Eritema pelas } \\
\text { Fraldas }\end{array}$ & Aplicar creme \\
\hline & & Avaliar eritema pelas fraldas \\
\hline Ferida & & Trocar a fralda \\
Cirúrgica & Ferida & Avaliar a ferida cirúrgica \\
& Cirúrgica & Executar tratamento da \\
& & ferida cirúrgica
\end{tabular}




\begin{tabular}{|c|c|c|}
\hline Focos & Diagnósticos & Intervenções \\
\hline & & Inserir dreno na ferida \\
\hline & & Remover dreno de ferida \\
\hline & & Remover sutura \\
\hline & & Trocar dreno de ferida \\
\hline & $\begin{array}{l}\text { Potencial para } \\
\text { melhorar o } \\
\text { conhecimento }\end{array}$ & $\begin{array}{l}\text { Avaliar conhecimento sobre } \\
\text { promoção da cicatrização da } \\
\text { ferida cirúrgica }\end{array}$ \\
\hline & $\begin{array}{l}\text { promoção da } \\
\text { cicatrização } \\
\text { da ferida } \\
\text { cirúrgica }\end{array}$ & $\begin{array}{l}\text { Ensinar sobre cuidados à } \\
\text { ferida cirúrgica }\end{array}$ \\
\hline \multirow{10}{*}{$\begin{array}{l}\text { Ferida } \\
\text { Traumática }\end{array}$} & \multirow{8}{*}{$\begin{array}{l}\text { Ferida } \\
\text { Traumática }\end{array}$} & Aplicar ligadura \\
\hline & & Avaliar ferida traumática \\
\hline & & $\begin{array}{l}\text { Executar tratamento da ferida } \\
\text { traumática }\end{array}$ \\
\hline & & Inserir dreno de ferida \\
\hline & & Remover dreno da ferida \\
\hline & & Remover sutura \\
\hline & & Suturar a ferida traumática \\
\hline & & Trocar dreno da ferida \\
\hline & \multirow{2}{*}{$\begin{array}{l}\text { Potencial para } \\
\text { melhorar o } \\
\text { conhecimento } \\
\text { sobre } \\
\text { promoção da } \\
\text { cicatrização } \\
\text { da ferida } \\
\text { traumática }\end{array}$} & $\begin{array}{l}\text { Avaliar conhecimento sobre } \\
\text { promoção da cicatrização da } \\
\text { ferida traumática }\end{array}$ \\
\hline & & $\begin{array}{l}\text { Ensinar sobre cuidados à } \\
\text { ferida traumática }\end{array}$ \\
\hline \multirow[t]{3}{*}{ Fissura } & \multirow[t]{3}{*}{ Fissura } & Aplicar creme \\
\hline & & Avaliar fissura \\
\hline & & $\begin{array}{l}\text { Executar tratamento da } \\
\text { fissura }\end{array}$ \\
\hline \multirow[t]{5}{*}{ Laceração } & \multirow[t]{5}{*}{ Laceração } & Avaliar laceração \\
\hline & & $\begin{array}{l}\text { Executar tratamento da } \\
\text { laceração }\end{array}$ \\
\hline & & Inserir dreno de ferida \\
\hline & & Remover dreno da ferida \\
\hline & & Remover sutura \\
\hline \multirow[t]{4}{*}{ Maceração } & \multirow[t]{4}{*}{ Maceração } & Aplicar creme \\
\hline & & Aplicar protetor cutâneo \\
\hline & & Avaliar a maceração \\
\hline & & $\begin{array}{l}\text { Executar tratamento da } \\
\text { maceração }\end{array}$ \\
\hline \multirow{4}{*}{$\begin{array}{l}\text { Membrana } \\
\text { Mucosa } \\
\text { Comprometida }\end{array}$} & \multirow{4}{*}{$\begin{array}{l}\text { Membrana } \\
\text { Mucosa } \\
\text { Comprometida }\end{array}$} & Aplicar creme \\
\hline & & $\begin{array}{l}\text { Avaliar o compromisso da } \\
\text { membrana mucosa }\end{array}$ \\
\hline & & $\begin{array}{l}\text { Executar o tratamento à } \\
\text { membrana mucosa }\end{array}$ \\
\hline & & Lavar a cavidade oral \\
\hline
\end{tabular}




\begin{tabular}{|c|c|c|}
\hline Focos & Diagnósticos & Intervenções \\
\hline \multirow[t]{3}{*}{ Queimadura } & \multirow[t]{3}{*}{ Queimadura } & Aplicar creme \\
\hline & & Avaliar queimadura \\
\hline & & $\begin{array}{l}\text { Executar tratamento da } \\
\text { queimadura }\end{array}$ \\
\hline \multirow[t]{4}{*}{ Úlcera Arterial } & \multirow[t]{2}{*}{ Úlcera Arterial } & Avaliar a úlcera arterial \\
\hline & & $\begin{array}{l}\text { Executar o tratamento } \mathrm{da} \\
\text { úlcera arterial }\end{array}$ \\
\hline & \multirow{2}{*}{$\begin{array}{l}\text { Potencial para } \\
\text { melhorar o } \\
\text { conhecimento } \\
\text { sobre } \\
\text { promoção da } \\
\text { cicatrização } \\
\text { da úlcera } \\
\text { arterial }\end{array}$} & $\begin{array}{l}\text { Avaliar o conhecimento sobre } \\
\text { promoção da cicatrização da } \\
\text { úlcera arterial }\end{array}$ \\
\hline & & $\begin{array}{l}\text { Ensinar sobre sinais de } \\
\text { complicações da úlcera } \\
\text { arterial }\end{array}$ \\
\hline \multirow{4}{*}{$\begin{array}{l}\text { Úlcera } \\
\text { Diabética }\end{array}$} & \multirow{4}{*}{$\begin{array}{l}\text { Úlcera } \\
\text { Diabética }\end{array}$} & Avaliar a úlcera diabética \\
\hline & & $\begin{array}{l}\text { Executar o tratamento da } \\
\text { úlcera diabética }\end{array}$ \\
\hline & & Inserir dreno de ferida \\
\hline & & Remover dreno da ferida \\
\hline \multirow[t]{6}{*}{$\begin{array}{l}\text { Úlcera } \quad \text { Por } \\
\text { Pressão }\end{array}$} & \multirow[t]{6}{*}{$\begin{array}{l}\text { Úlcera } \quad \text { Por } \\
\text { Pressão }\end{array}$} & $\begin{array}{lll}\text { Aliviar a pressão } & \text { com } \\
\text { dispositivos } & & \end{array}$ \\
\hline & & Aplicar creme \\
\hline & & Avaliar a úlcera por pressão \\
\hline & & $\begin{array}{l}\text { Executar o tratamento da } \\
\text { úlcera por pressão }\end{array}$ \\
\hline & & Inserir dreno de ferida \\
\hline & & Remover dreno da ferida \\
\hline \multirow[t]{6}{*}{ Úlcera Venosa } & \multirow[t]{6}{*}{ Úlcera Venosa } & Aplicar terapia compressiva \\
\hline & & Aplicar ligadura elástica \\
\hline & & Avaliar úlcera venosa \\
\hline & & $\begin{array}{l}\text { Executar tratamento } \\
\text { úlcera venosa }\end{array}$ \\
\hline & & Inserir dreno de ferida \\
\hline & & Remover dreno da ferida \\
\hline
\end{tabular}

\subsection{Fase 2}

Foi realizada em abril de 2019 uma reunião com o grupo focal na ESEP com quatorze enfermeiros membros do CIDESI previamente contactados por e-mail. Foram convidados pela sua experiência com terminologias de enfermagem e várias contribuições para os projetos de investigação anteriores relacionados com os SIS. Todos os enfermeiros possuem o grau de mestre e mais de metade o doutoramento em Ciências de Enfermagem. Este encontro durou cerca de 120 minutos e foi gravado o áudio após a obtenção do consentimento de todos os participantes.

O investigador principal, doutorando em ciências de enfermagem, com o grau de mestre, assumiu o papel de facilitador. Este investigador teve várias formações e experiências prévias com investigação qualitativa. 
Foram explicados os objetivos do projeto e de seguida cada diagnóstico, intervenção e relação foram apresentados individualmente, pedindo uma opinião relativa à categorização ou exclusão realizada anteriormente pelos investigadores. Todas as categorias apresentadas foram aceites pelo grupo, com exceção de dois assuntos.

Sete intervenções relacionadas com o autocuidado: posicionar-se, como "Assistir a pessoa a posicionar-se" e "Ensinar a sobre posicionamento no leito", foram excluídas durante a análise de conteúdo por se focarem no compromisso da pessoa se posicionar no leito e não na cicatrização da ferida. No entanto, foi discutido que a presença da ferida por alterar a forma como o utente se posiciona ou é posicionado para promover esta cicatrização. Foram criadas categorias para acomodar as recomendações do grupo focal.

Três intervenções relacionadas com as ostomias intestinais ("Avaliar a pele periférica ao estoma", "Trocar saco de ostomia" e "Otimizar dispositivos de ostomia") foram inicialmente excluídas por se relacionarem com os diagnósticos de ostomias (por exemplo "Potencial para melhora a habilidade para trocar o dispositivo de ostomia"). O grupo focal sugeriu que a presença de uma ferida, como "Maceração", pode alterar a forma como o dispositivo é aplicado ou o próprio tipo de dispositivo.

O último assunto discutido na reunião foi a reintrodução de problemas previamente corrigidos com a proposta da parametrização única de 2014. Como os peritos envolvidos nesta proposta também estiveram presentes no grupo focal, levantou-se a questão sobre como estes problemas voltaram a surgir no SIS, contradizendo as opiniões anteriores de peritos (Paiva et al., 2014). Um exemplo destes problemas foi a não existência dos diagnósticos focados no conhecimento para cada achado clínico (como o Potencial para melhorar o conhecimento sobre promoção da cicatrização da queimadura). Outro exemplo foi a associação de intervenções do tipo ensinar nos próprios diagnósticos de feridas, em vez dos diagnósticos como foco no conhecimento.

\section{Discussão}

Com os dados extraídos e analisados da parametrização do SIS, não podemos continuar a concluir que existe a falta de padronização na linguagem, como referido por Gonçalves et al. (2019), e Neves e Parente (2019) ao analisar uma geração anterior a 2014. O número limitado de frases por categoria demonstra uma abordagem mais focada ao documentar os diagnósticos e intervenções no tratamento de feridas, atribuindo os mesmos nomes aos mesmos conceitos.

O critério de exclusão mais frequente foi a associação a outros focos não tegumentares, mas que podem contribuir para a ocorrência de um diagnóstico de ferida. A prevenção destes diagnósticos, como úlceras por pressão, são uma área significativa dos cuidados de enfermagem. No entanto, os SIS e os modelos de informação dependem de uma estrutura com o mínimo de redundância possível numa sequência conceptual e lógica no processo de tomada de decisão.

Seria expectável encontrar um diagnóstico centrado no conhecimento por cada foco, como - "Potencial para melhorar o conhecimento sobre promoção da cicatrização da úlcera arterial". Não conseguimos concluir sobre o motivo para isto não ter acontecido, dado que estes diagnósticos existiam na proposta da parametrização única (Paiva et al., 2014). Estas alterações contradizem as opiniões de peritos anteriores e foram mencionadas como "problemas" no grupo focal.

Com a introdução do nível regulatório da parceria com a Ordem dos Enfermeiros (2018) no desenvolvimento da ontologia, esperamos que todas as atualizações nos conteúdos do SIS evoluam para decisões suportadas em evidência clínica.

A promoção da cicatrização de feridas envolve uma abordagem dirigida à pessoa com a prescrição de um tratamento seguro, efetivo e apropriado (Lumbers, 2018). Algumas relações inadequadas entre diagnósticos e intervenções foram encontradas. Enquanto os SIS forem utilizados para registos descritivos após os cuidados realizados, estas relações não trazem consequências para a pessoa. 
No entanto, quando os sistemas evoluírem para um apoio à tomada de decisão clínica, a sugestão de intervenções inadequadas para os dados avaliados pode conduzir a dano físico, o que nos leva de volta à questão da responsabilidade pela criação, manutenção e validação dos conteúdos para os SIS.

Os modelos clínicos não têm por objetivo substituir os profissionais de saúde, mas são desenhados para promover uma tomada de decisão sustentada em evidência (Bezemer et al., 2019). A versão inicial desde modelo de dados centrado na cicatrização de feridas promove melhores decisões ao assegurar que os mesmos conceitos clínicos são usados com as mesmas palavras e frases no mesmo contexto. Também contribui para a continuidade de cuidados, essencial para prevenir a fragmentação dos cuidados na passagem da pessoa por várias instituições com vários sistemas em uso (Bahr \& Weiss, 2019).

\section{Conclusões}

O modelo de diagnósticos e intervenções de enfermagem mostra uma dimensão clínica alargada na área de intervenção autónoma relacionada com o sistema tegumentar. A documentação continua a migrar de notas descritivas para um processo organizado com diagnósticos e intervenções consistentes estruturados com uma linguagem padronizada internacional.

A abordagem qualitativa dos métodos utilizados permitiu uma contribuição significativa para a interoperabilidade semântica entre sistemas de informação, progredindo quer em termos dos conceitos utilizados para representar a prática clínica, quer na validade da construção dos enunciados que representam os diagnósticos de enfermagem as intervenções implementadas.

Recomendamos que investigações futuras se centrem em artigos científicos revistos por pares de forma a extrair todos os diagnósticos, intervenções e dados de avaliação com evidência clínica para suportar os achados descritos neste artigo. O modelo aqui apresentado corresponde à primeira etapa na construção de um modelo clínico de informação centrado na cicatrização de feridas.

\section{Referências}

Atkin, L., \& Tickle, J. (2018). Best practice statement leg ulceration pathway: revision required to reflect new evidence. Wounds UK, 14(4).

Bahr, S. J., \& Weiss, M. E. (2019). Clarifying model for continuity of care: a concept analysis. International journal of nursing practice, 25(2), e12704.

Bardin, L. (1977). Análise de conteúdo (Vol. 70).

Batista da Cunha, J., Alves Dutra, R. A., Magela Salomé, G., \& Masako Ferreira, L. (2018). Computational system applied to mobile technology for evaluation and treatment of wounds. Revista de Enfermagem UFPE, 12(5). https://doi.org/https://doi.org/10.5205/1981-8963V12I5A230677P1263-1272-2018

Bezemer, T., De Groot, M. C., Blasse, E., Ten Berg, M. J., Kappen, T. H., Bredenoord, A. L., Van Solinge, W. W., Hoefer, I. E., \& Haitjema, S. (2019). A human (e) factor in clinical decision support systems. Journal of medical Internet research, 21(3), e11732. https://doi.org/10.2196/11732

Cruz, I., Bastos, F., Pereira, F., Silva, A., \& Sousa, P. (2016). Analysis of the Nursing Documentation in-Building a Clinical Data Use in Portugal Model of Nursing Centered on the Management of Treatment Regimen. Nursing Informatics 2016: EHealth for All: Every Level Collaboration-From Project to Realization, 225, 407. https://doi.org/10.3233/978-1-61499658-3-407

European Observatory on Health Systems and Policies. (2018). Health System Review - Portugal, Phase1 Final Report. https://www.sns.gov.pt/wpcontent/uploads/2018/04/PortugalReviewReport_Printers_03April2018-2.pdf 
Gonçalves, P., Sequeira, C., \& Silva, M. (2019). Content analysis of nursing diagnoses in menta health records in Portugal. International nursing review, 66(2), 199-208.

https://doi.org/10.1111/inr.12493

Gruber, T. (1993). What is an Ontology.

International Council of Nurses. (2017). International Classification for Nursing Practice. Retrieved 2021/01/23 from https://www.icn.ch/what-we-do/projects/ehealth-icnptm/icnp-browser

International Organization for Standardization. (2014). Health informatics - Categorial structures for representation of nursing diagnoses and nursing actions in terminological systems (ISO 18104).

International Organization for Standardization. (2015). Health informatics - Detailed clinical models, characteristics and processes (ISO/TS 13972:2015).

Itulua-Abumere, F. (2015). Outlines of Focus Group: Exploring the potentials of focus group research method/ perspective for practical research purposes whilst indicating the limitations and advantages of the method. https://doi.org/10.13140/RG.2.1.4509.8966

Ivins, N., Clask, M., \& Fallon, M. (2018). An initiative to improve wound management within community services across one Clinical Commissioning Group in England. Wounds UK, 14(5).

Lindholm, C., \& Searle, R. (2016). Wound management for the 21 st century: combining effectiveness and efficiency. International wound journal, 13, 5-15. https://doi.org/doi.org/10.1111/iwj.12623

Lozano-Rubí, R., Carrero, A. M., Balazote, P. S., \& Pastor, X. (2016). OntoCR: A CEN/ISO-13606 clinical repository based on ontologies. Journal of biomedical informatics, 60, 224-233. https://doi.org/https://doi.org/10.1016/j.jbi.2016.02.007

Lumbers, M. (2018). Wound debridement: choices and practice. British Journal of Nursing, 27(15), S16-S20. https://doi.org/10.12968/bjon.2018.27.15.s16

Neves, H., \& Parente, P. (2019). A nursing clinical data model for neuromuscular processes: content analysis of the Portuguese nursing customization. Ciencia \& saude coletiva, 24, 1609 1616. https://doi.org/10.1590/1413-81232018245.04462019

Nussbaum, S. R., Carter, M. J., Fife, C. E., DaVanzo, J., Haught, R., Nusgart, M., \& Cartwright, D. (2018). An economic evaluation of the impact, cost, and medicare policy implications of chronic nonhealing wounds. Value in Health, 21(1), 27-32. https://doi.org/10.1016/j.jval.2017.07.007

Ordem dos Enfermeiros. (2018). OE celebra parceria com Escola Superior de Enfermagem do Porto. Retrieved 2021/01/21 from https://www.ordemenfermeiros.pt/noticias/conteudos/oecelebra-parceria-com-escola-superior-de-enfermagem-do-porto/

Paiva, A., Cardoso, A., Sequeira, C., Morais, E., Bastos, F., \& Pereira, F. (2014). Análise da parametrização nacional do Sistema de Apoio à Prática de Enfermagem-SAPE®. Escola Superior de Enfermagem do Porto, Porto. http://hdl.handle.net/10400.26/31982

Queirós, C., Silva, M. A. T. C. P., Cruz, I., Cardoso, A., \& Morais, E. J. (2021). Nursing diagnoses focused on universal self-care requisites. International nursing review. https://doi.org/10.1111/inr.12654

Samuriwo, R., \& Dowding, D. (2014). Nurses' pressure ulcer related judgements and decisions in clinical practice: a systematic review. International journal of nursing studies, 51(12), 1667 1685. https://doi.org/10.1016/j.ijnurstu.2014.04.009

Strickler, J. (2017). Florence Nightingale: Lighting the way for the future of nursing. Nursing, 47(12), 43-45. https://doi.org/10.1097/01.nurse.0000526887.95058.3b

WOCN Task Force. (2018). Wound, Ostomy, and Continence Nursing: Scope and Standards of WOC Practice, 2nd Edition: An Executive Summary. J Wound Ostomy Continence Nurs, 45(4), 369-387. https://doi.org/10.1097/WON.0000000000000438

Woo, K., Conceição de Gouveia Santos, V. L., \& Alam, T. (2018). Optimising quality of life for people with non-healing wounds. Wounds International, 9(3), 6-14. http://search.ebscohost.com/login.aspx?direct=true\&db=ccm\&AN=131918860\&lang=ptbr\&site=ehost-live

World Health Organization. (2017, 2017/07/04). International Classification for Nursing Practice (ICNP). Retrieved 2020/02/18 from https://www.who.int/classifications/icd/adaptations/icnp/en/ 